Research Article

\title{
Mode-I Metal-Composite Interface Fracture Testing for Fibre Metal Laminates
}

\author{
Periyasamy Manikandan (D) and Gin Boay Chai (i) \\ Aerospace Engineering Cluster, School of Mechanical and Aerospace Engineering, Nanyang Technological University, Singapore \\ Correspondence should be addressed to Gin Boay Chai; mgbchai@ntu.edu.sg
}

Received 15 October 2017; Accepted 20 December 2017; Published 28 February 2018

Academic Editor: Fabrizio Sarasini

Copyright () 2018 Periyasamy Manikandan and Gin Boay Chai. This is an open access article distributed under the Creative Commons Attribution License, which permits unrestricted use, distribution, and reproduction in any medium, provided the original work is properly cited.

\begin{abstract}
The main contribution of the present paper is the determination of the mode-I fracture of metal-composite interface region for fibre metal laminates (FMLs). A hybrid DCB configuration is proposed to investigate the mode-I fracture between metalcomposite interface using experimental and numerical approaches. A computationally efficient and reliable finite element model was developed to account for the influence of metal plasticity on the measured fracture energy. The results of the experimental and numerical studies showed that metal plasticity increases the fracture energy of the metal-composite interface as the fracture event progresses. The applied energy truly utilized to propagate metal-composite interface fracture was predicted numerically by extracting the elastic strain energy data. The predicted true fracture energy was found to be approximately $50 \%$ smaller than the experimentally measured average propagation energy. The study concluded that metal plasticity in hybrid DCB configuration overpredicted the experimentally measured fracture energy, and this can be alleviated through numerical methodology such as the finite element approach as presented in this paper.
\end{abstract}

\section{Introduction}

Hybridization of material not only makes it structurally efficient but also makes it multifunctional. With sustained research and advancements in adhesive bonding technology, designers are able to successfully develop a novel metalbonded hybrid composite structure such as the fibre metal laminates (FMLs) in the aviation industry. Currently, FML structures are the third largest primary load-carrying aviation material by volume housing nearly $470 \mathrm{sq} \cdot \mathrm{m}$ of the upper fuselage section of Airbus A380 passenger air carrier [1]. FML is a versatile and high performance hybrid material made up of alternating layers of thin metal sheets and thin fibre reinforce polymeric (FRP) composite layers. Compounding metal and composite makes FML stronger, lighter, better damage tolerant, good fatigue resistant, better corrosion, and impact resistant material which is not feasible through single material alone $[2,3]$.

The effectiveness of FML as a good load-carrying material relies heavily on the level of the bonding integrity between the metal and the FRP composite layers [4]. The bonding interface must possess sufficient interfacial strength to be able to distribute the load between two characteristically different materials. There are two different bonding interfaces in a traditional FML: (i) adhesive-metal and (ii) adhesive-composite. The level of bonding integrity is strictly dependent on the appropriate surface pretreatment of bonded metal to composite interfaces and the chemical compatibility of adhesive introduced. Apart from those, the potential bonding strength is also dependent on the stiffness imbalance and the thermal mismatch of dissimilar materials during testing and curing, respectively [5]. For the case of FML, the problem of thermal mismatch is a challenging one where the difference in thermal expansion coefficients between adhesive, metal, and composite layers needs to be resolved. A study [6] had shown that poststretching after curing helps to enhance delamination resistance in the FML panels.

Adhesive bonding between monolithic material substrates like metal-metal and composite-composite is fairly 
well understood, and the testing procedures to evaluate their fracture properties are fairly standardized. However, this is not the case for evaluating dissimilar material joints like metal-composite interface. Furthermore, plastic yielding is predominately sensitive in the DCB sample having thin metal adherent and may attenuate the crack propagation in fracture test thereafter [7]. Such plasticity can result in abnormally high values of fracture toughness, and it is necessary to account for its influence in order to obtain the true magnitude of the fracture energy. So far in literature, researchers had employed three different approaches to account for the plasticity effects: (i) experimental approach using thick metal doublers to attenuate plastic flow $[6,8]$, (ii) theoretical approach [9], and (iii) finite element approach [7]. In the last two approaches, efforts were made to estimate the fraction of plastically absorbed energy from the overall internal strain energy.

Reeder et al. [8] portrayed the method of bonding doublers to thin substrates in a manner to avoid plastic deformation or premature adherent failure due to bending before the interface delamination initiate. The standard Irwin-Kies fracture energy equation was revised for the samples having bonded doublers for all three modes of fracture. Vlot and Van Ingen [6] utilized a similar concept to evaluate the delamination fracture energy of metal-composite interface of FML. To achieve stable delamination propagation in the mode-I test using the double cantilever beam (DCB) geometry, the transverse displacement of loading platen was stopped at every interval when there exists a delamination growth and stayed until the load magnitude leveled off. Lawcock et al. [10] followed the standard testing procedure of metallic joints to evaluate mode-I fracture energy of FML assuming that the volume fraction of the composite in the considered DCB geometry is negligibly small compared to the metal volume.

Reyes et al. [11] evaluated the mode-I, mode-II, and mixed-mode fracture energy of metal-composite interface of FML using a single cantilever beam (SCB), a double end notched flexure, and a modified mixed-mode flexure geometry, respectively. Using the SCB geometry, stable interface crack propagation without causing plastic yielding of metal sheet would become feasible. However, if the fracture energy of the adhesive material is far larger than the yielding stress of the metal sheet, this method could enforce more challenge to initiate interface crack growth.

The aim of the present paper is to investigate the mode-I fracture energy evaluation of metal-composite joints in order to understand the interface crack growth in FML that involves the influence of the plastic yielding in thin metal sheets. Finite element approach was utilized to estimate the amount of energy dissipated through plastic deformation. The robustness of the developed numerical model was validated by comparing the fracture test results of monolithic composite-composite sample with those obtained from the numerical study. Such comparison also explores the influence of plasticity effects and allows one to quantitatively estimate how far the fracture energy magnitude has been altered due to such additional irreversible deformation.

\section{Materials and Test Methodology}

In this section, the mode-I fracture test geometry and the methodology used in the study are described. Subsequently, the investigation of mode-I fracture behavior of compositecomposite and metal-composite joints are presented, and some conclusive remarks are narrated in the final section.

2.1. Materials. Aluminum alloy Al 2024-0, L-530 8-harness satin weave 7781 glass epoxy prepreg, and Redux $335 \mathrm{~K}$ adhesive film are the materials used to fabricate the adhesively bonded fibre-metal laminated samples investigated in this study. Before bonding, the aluminum alloy bonded surfaces were abraded using 320 grit size sand paper, cleaned with acetone solvent, and appropriately surface treated in a motive to promote good chemical linkage between aluminum-adhesive-composite interface.

Metal-composite layers of designated stacking were laminated and bonded with the single layer of adhesive film. Stacked laminates were cured in accordance with the composite manufacturer data sheet using an autoclave chamber under 3 bar and $120^{\circ} \mathrm{C}$ in the presence of vacuum. The cured laminates were cut to the required dimension without disturbing the adhesively bonded interface using a high-speed water jet cutter, and quality checks were carefully carried out under the optical microscope.

2.2. Experimental Tests. In total, three different configurations of DCB tests were experimented to investigate the mode-I fracture behavior of metal-composite adhesive interface fracture growth. They are as follows:

(i) Thick metal doublers bonded with thin composite adherent, $M-C-M$ configuration

(ii) Monolithic composite adherents with in situ epoxy matrix adhesive interface, $C$ - $C$ configuration

(iii) Hybrid geometry with firmly bonded metalcomposite adherent as one arm and monolithic composite adherent as another arm, $C-M-C$ configuration

Schematic representation of above listed configurations and relevant dimensions are shown in Figures 1(a)-1(c), respectively. Samples were tested at a rate of $3 \mathrm{~mm} / \mathrm{min}$ using a universal testing machine. Magnitude of load $P$, cross head displacement $\delta$, and crack length $a$ were recorded during both crack initiation and instant propagation.

The fracture energy for M-C-M configuration is evaluated using the following guidelines of ASTM D3833 assuming that the composite layer is very thin compared to thick aluminum doublers [10]. The equation of mode-I fracture energy, $G_{I}$, is given by

$$
G_{I}=\frac{4 P^{2}}{E B^{2} t_{1}^{2}}\left[3 a_{o}^{2}+t_{1}^{2}\right],
$$

where $E, B$, and $t_{1}$ are Young's modulus, width, and thickness of the aluminum adherends. 


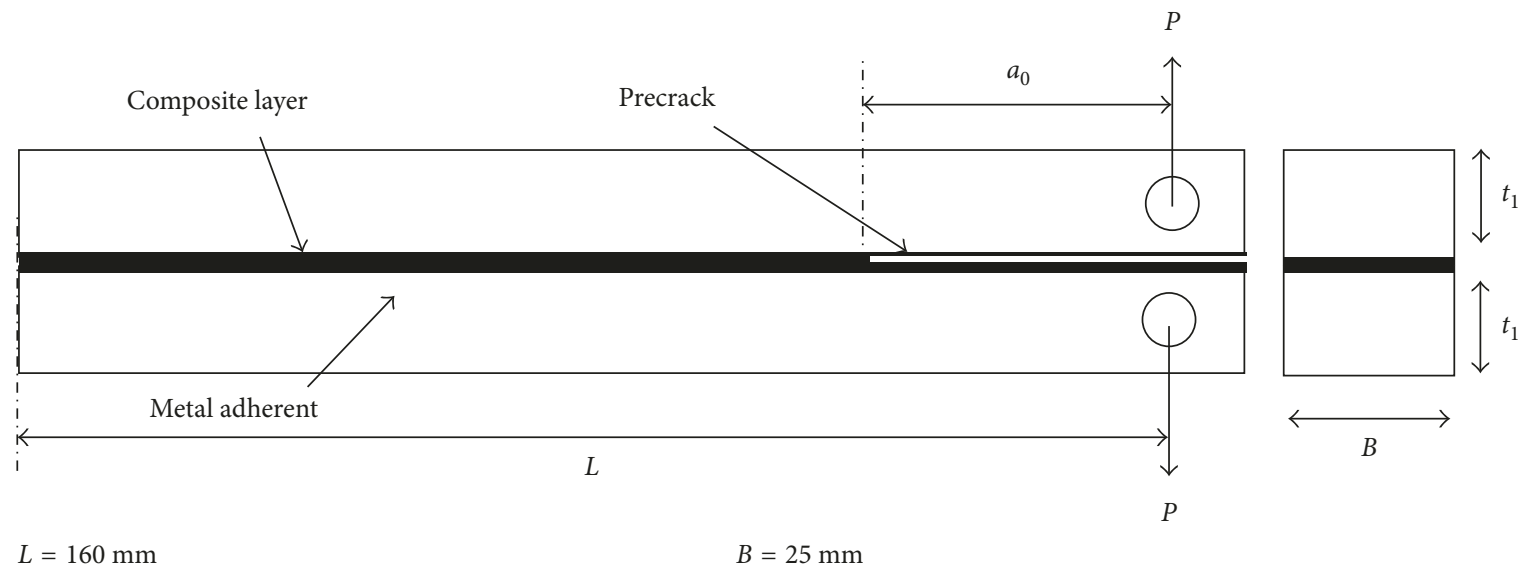

$$
a_{0}=45 \mathrm{~mm}
$$$$
t_{1}=10 \mathrm{~mm}
$$

(a)

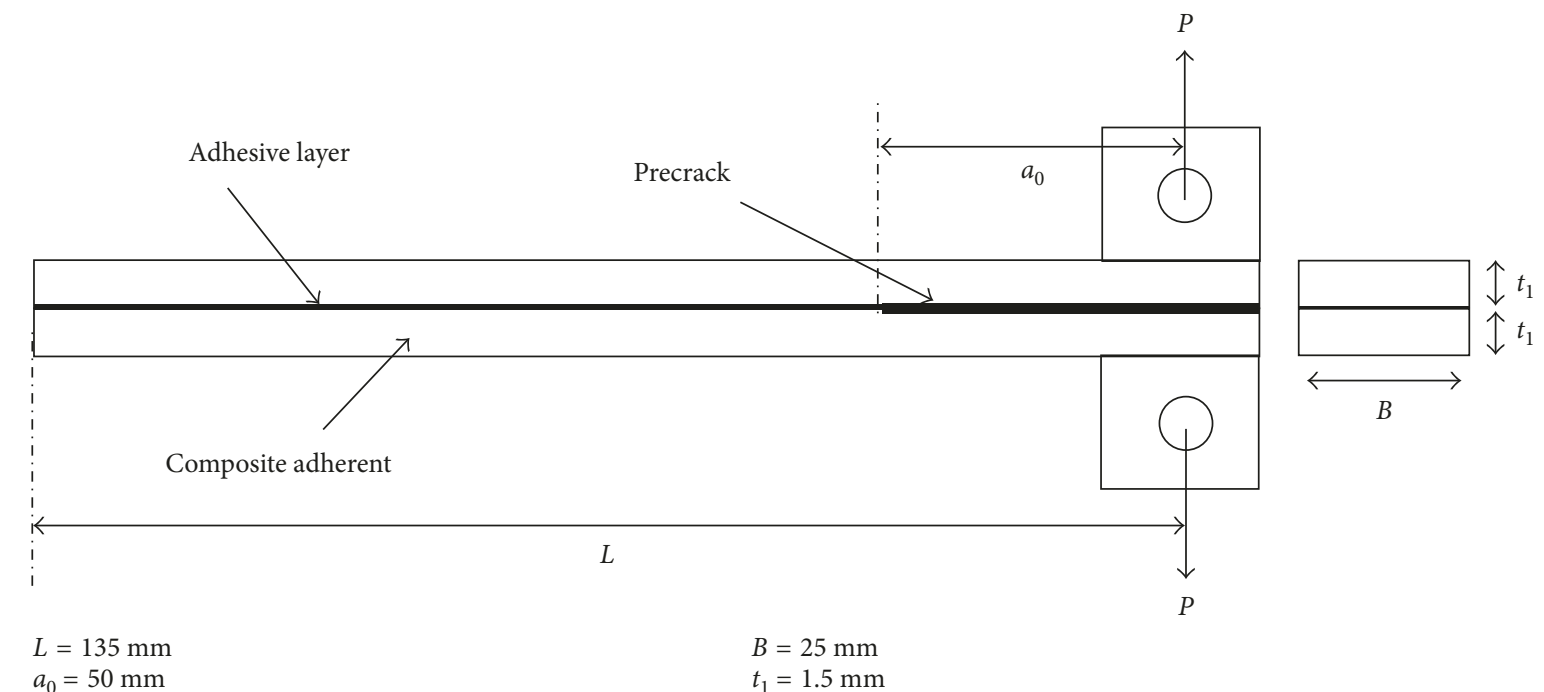

(b)

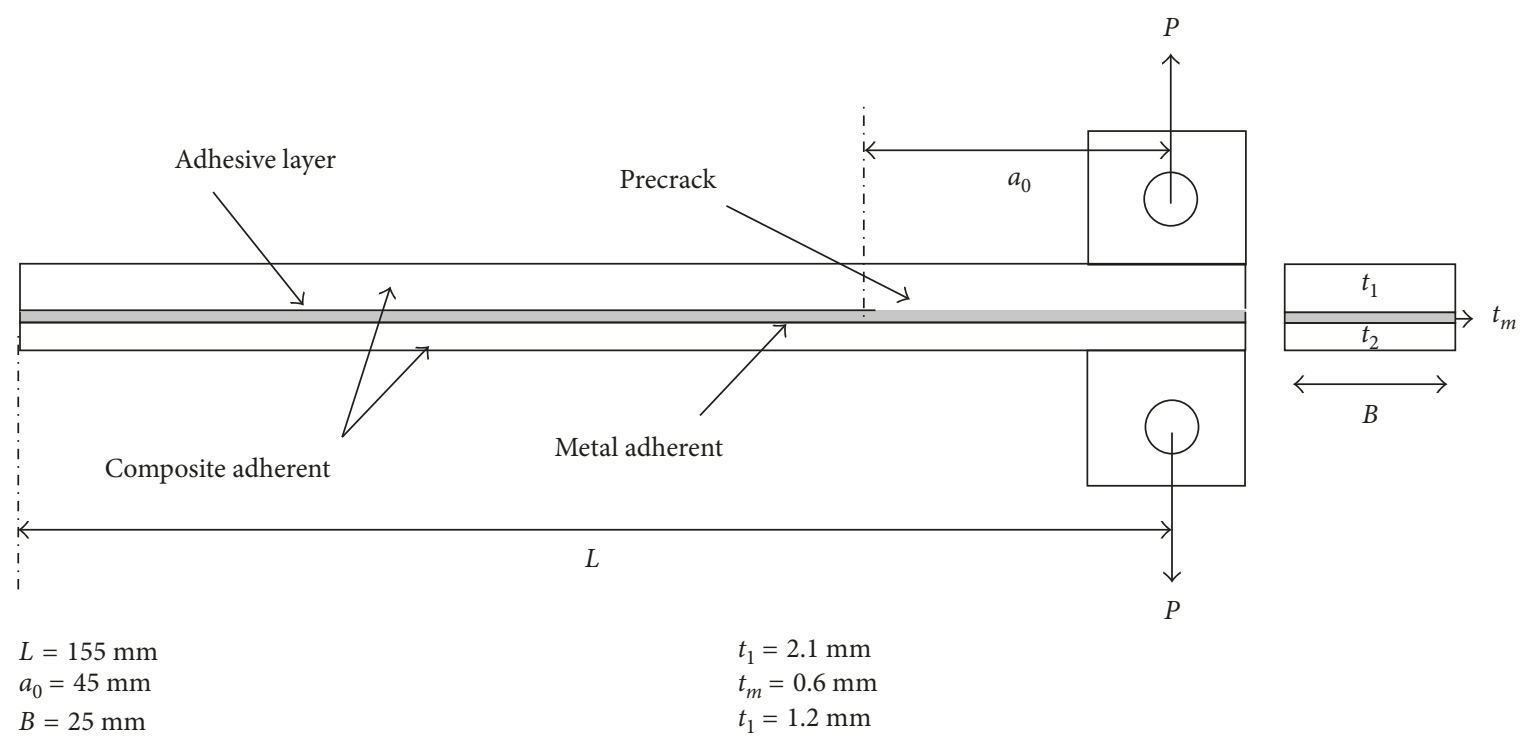

(c)

FIGURE 1: Schematic representation of DCB geometry: (a) M-C-M configuration, (b) C-C configuration, and (c) C-M-C configuration. 


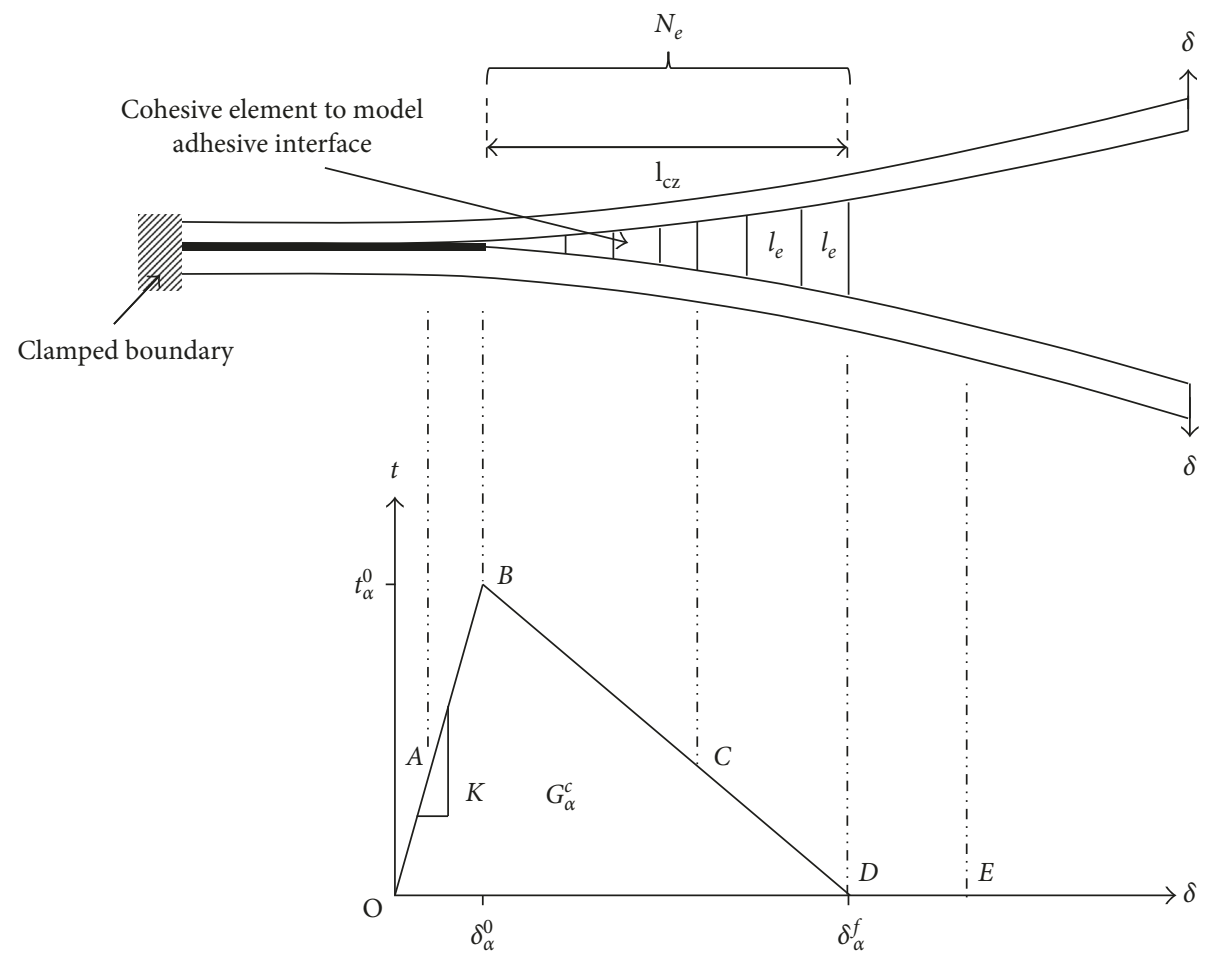

FIgURE 2: Bilinear traction-separation law for cohesive elements in the DCB model.

While for the C-C and C-M-C configurations, the value of fracture energy is ascertained using the Irwin-Kies equation [12] given by

$$
G_{I}=\frac{P^{2}}{2 B} \cdot \frac{d C}{d a},
$$

where $C=\delta / P$ is the specimen compliance and $a$ is the corresponding crack length for a given displacement of $\delta$.

2.3. Finite Element Simulation. The simulation of interface crack initiation and propagation was performed using cohesive element in the commercial finite element platform, ABAQUS/Standard. The constitutive formulation of cohesive element is modeled based on the traction $(t)$ separation $(\delta)$ law that relates the crack opening displacement in the process zone to the resisting tractions [13]. The material model of cohesive element is defined by an initial elastic stiffness, $\mathrm{K}$, peak traction which causes crack initiation, $t_{\alpha}^{0}$, and critical energy release rate equal to area under tractionseparation curve responsible to cause crack-propagation rate, $G_{\alpha}^{c}$. The schematic of the bilinear traction-separation law used to simulate the progressive interface crack of DCB configuration in this paper is shown in Figure 2.

The input parameters required to model the cohesive elements are determined based on Turon's methodology [14]. The methodology provides a systematic procedure to calculate the length of the cohesive zone, $l_{\mathrm{cz}}$, size of the cohesive element, $l_{e}$, and number of cohesive elements, $N_{e}$, required to model the fracture growth accurately. The methodology also allows us to relax the requirement of extremely fine meshes through artificially increasing the length of cohesive zone by reducing the peak traction, $t_{\alpha}^{0}$, magnitude. The mathematical derivations to obtain adjusted traction strength, $t_{\alpha}^{a}$, and the optimum mesh parameters using Turon's methodology are summarized in the Appendix. Both metal and composite adherents are modeled using isotropic and orthotropic elastic material properties, respectively. These layers are discretized using an incompatible-mode eight-node brick element, C3D8I, and the interface region using three-dimensional cohesive element, COH3D8.

The material properties used in the finite element model for the substrates and interface region are provided in Table 1. The FE model was validated by comparing the predicted load-displacement results with the experimental results. Using this curve, fracture energy data are calculated by employing the area method given by [15]

$$
G_{I}=\frac{\Delta U}{B \Delta a},
$$

where $\Delta U$ is the area under numerical $P-\delta$ curve between consecutive crack growth intervals and $\Delta a$ is the corresponding crack length increment as shown in Figure 3.

\section{Results and Discussion}

3.1. Selection of DCB Configuration. The initial emphasis to determine the metal-composite fracture behavior was analyzed through the experimental study of the M-C-M configuration. Enhanced resistance to bending because of thick aluminum adherends resulting in multiple interfacial and interlaminar crack propagation was observed between $\mathrm{M}-\mathrm{C}$ and $\mathrm{C}-\mathrm{C}$ regions, respectively, as shown in Figure 4(a). 
TABLE 1: Material properties used in the FE model.

\begin{tabular}{lcc}
\hline Response & Property & Value \\
\hline \multirow{2}{*}{ Metal } & Density & $\rho=2780 \mathrm{~kg} / \mathrm{m}^{3}$ \\
& Young's modulus & $E=70 \mathrm{GPa}$ \\
$\nu=0.3$ & $\rho=1900 \mathrm{~kg} / \mathrm{m}^{3}$ \\
& Poisson's ratio & $E_{1+}=E_{2+}=24 \mathrm{GPa}$ \\
Composite & Density & $E_{1-}=E_{2-}=24 \mathrm{GPa}$ \\
& In-plane tensile Young's modulus & $G_{12}=3.6 \mathrm{GPa}$ \\
& In-plane compression Young's modulus & $\nu_{12}=0.1$ \\
Adhesive & Shear modulus & $K_{\mathrm{nn}}=K_{\mathrm{ss}}=K_{\mathrm{tt}}=1 e^{15} \mathrm{~N} / \mathrm{m}^{3}$ \\
& Poisson's ratio & $t_{n}^{0}=t_{s}^{0}=t_{t}^{0}=50 \mathrm{MPa}$ \\
\hline
\end{tabular}

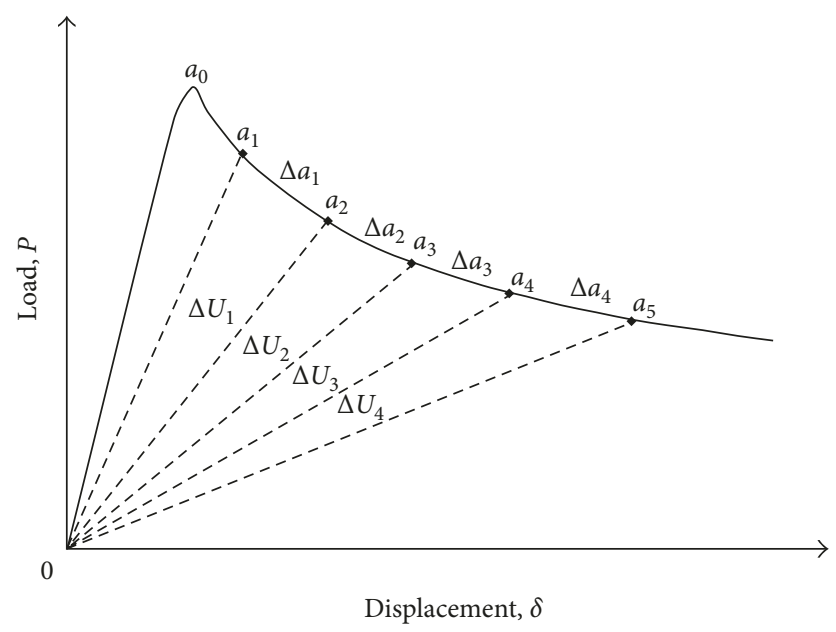

Figure 3: Area method of fracture energy determination.

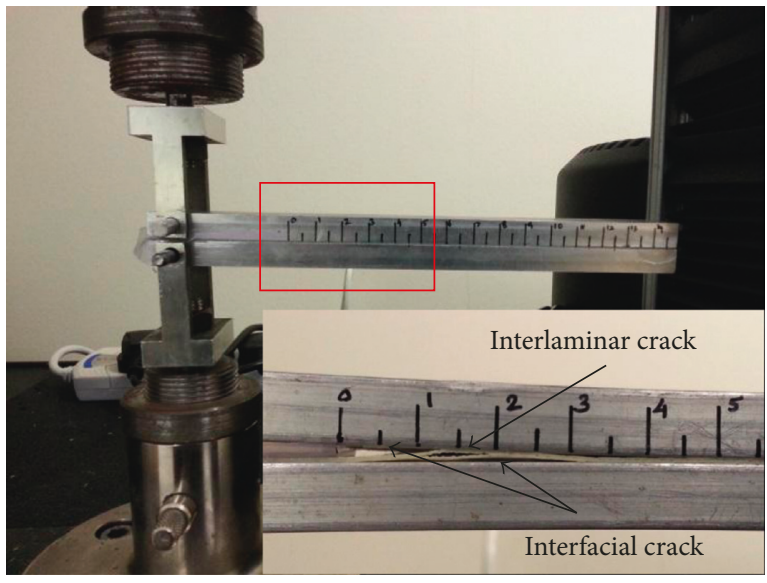

(a)

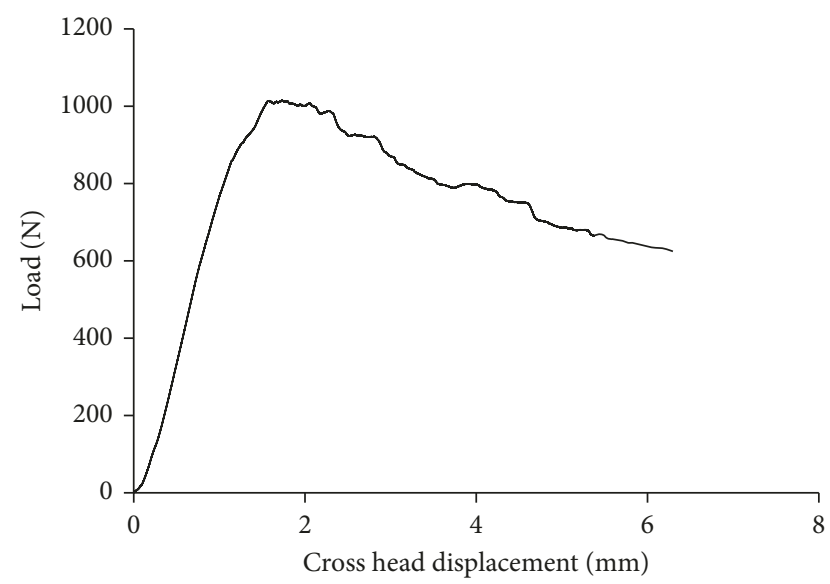

(b)

Figure 4: M-C-M DCB configuration: (a) multiple interface crack surfaces and (b) typical load-displacement curve.

Despite such multiple crack nucleation scenarios, the load variations associated with those multiple interface crack propagation do not show any discrete load drops as illustrated in Figure 4(b). This behavior indicates that the presence of thick aluminum adherends desensitized these multiple surface nucleation. Only the initiation fracture energy is feasible from this experiment using (1) which amounted to about $0.656 \mathrm{~N} / \mathrm{mm}$.

The DCB geometry with dissimilar adherends having bimaterial metal-composite adherent as one arm and monolithic composite as another arm (C-M-C configuration) was found to be a feasible design that allows the 
evaluation of the fracture energy not only in the crack initiation phase but also throughout crack propagation path. Thin adherends admit the arm to bend freely and facilitate the preimitated crack front propagation along metalcomposite interface in stable manner even though there exist a significant amount of plastic deformation in the thin metal layer.

For the case of the hybrid configuration of DCB arms, the bending centroids of the two arms are generally different, and this difference results in unsymmetric flexure and inherently induces the mixed-mode loading state on the precrack front instead of pure mode-I loading state. Such mixed-mode inference was attenuated by selecting appropriate thickness for two DCB arms in a manner to have equal bending rigidity, EI, for both adherent arms. The derived geometric dimensions of the C-M-C configured DCB design is given in Figure 1(c).

3.2. DCB Results of C-C Configuration. The DCB test using the $\mathrm{C}-\mathrm{C}$ configuration is performed to obtain an accurate fracture energy of adhesive interface where the adherents undergo only elastic deformation. This study primarily serves as a benchmark that allows one to understand the metal sheet plastic deformation influence on $G_{I}$ magnitude in the C-M-C configuration test. Moreover, it also provides opportunity to check the reliability of developed finite element model by ensuring that the internal energy utilized to delete the cohesive interface elements is the same as that of the experimentally predicted $G_{I}$.

The experimental and predicted mode-I fracture crack interface between composite adherends is shown in Figures 5(a) and 5(b), respectively. No visible fibre bridging patterns were found along the cracked interface. This is because the weave pattern of the woven composite adherends completely hindered the fibre pullout failure. Figure 6 illustrates the comparison plots of the load $P$ versus deflection $\delta$ and the fracture energy $G_{I}$ versus crack extension $\Delta a$. The smooth experimental load variation with no discrete load drops and change in fracture energy attributed in propagation phase confirms that the crack growth surfaces are pure cohesive in nature as shown in Figure 5(a). The average experimental fracture energy to cause crack initiation is found to be about $0.2 \mathrm{~N} / \mathrm{mm}$, and it increased to about $0.65 \mathrm{~N} / \mathrm{mm}$ and maintained constant throughout the crack propagation phase. The latter propagation phase $G_{I}$ is used as a damage evolution material data input for cohesive elements in finite element simulation.

3.3. Selection of Optimum Mesh Parameters. The optimum mesh parameters required to simulate DCB mode-I fracture were found using the equations presented in the Appendix. The minimum cohesive zone length required to model mode-I fracture based on the experimental fracture energy for the C-C configuration is estimated about $0.29 \mathrm{~mm}$ using (A.1), and the material properties used are listed in Table 1. Based on that magnitude, parametric studies for three levels of mesh $l_{e}\left(\geq l_{\mathrm{cz}}\right)$ were conducted for four different $N_{e}$ in the cohesive zone length. The corresponding magnitude of adjusted traction strengths and artificially increased cohesive zone length for crack propagation was found using (A.3) and (A.1), respectively, and these are tabulated in Table 2.

The predicted load-deflection response for different levels of mesh refinement is shown in Figure 7. Irrespective of $l_{e}$, the response predicted by the adjusted interfacial strength $\bar{t}_{\alpha}^{a}$ with $N_{e}=1$ did not converge and no numerical solution exists. For all other cases, the predicted softening crack propagation response is almost similar compared to the experimental measurement with some minor variations.

For the mesh size of $l_{e}=1 \mathrm{~mm}$, the softening response shows spurious oscillations for smaller $N_{e}$ which means the mesh size is too coarse to accurately predict the crack propagation. Take note that the amplitude of the spurious oscillation is found to be attenuated with increase in $N_{e}$. It can be seen that the responses obtained with the finer mesh size of $l_{e}=0.5 \mathrm{~mm}$ and $l_{e}=0.3 \mathrm{~mm}$ overpredicted the peak load. Meanwhile for this mesh size, the softening response exhibited smooth crack propagation. On comparing the number of iterations required to complete the solution, mesh sizes with more $N_{e}$ in its artificially increased cohesive zone length $l_{\mathrm{cz}}$ have shown to be computationally efficient.

The above comparative study confirms that the interface strength $\bar{t}_{\alpha}^{0}$ required to initiate crack propagation does not have a significant influence on the overall response. Thus, the interface strength modification and the artificial cohesive zone length increment strategy initially proposed by Turon [14] can be used to model accurate crack propagation using cohesive elements at coarse mesh level. For the present DCB simulation, results obtained using lowest adjusted interfacial strength $\bar{t}_{\alpha}^{a}$ with larger $N_{e}\left(\mathrm{l}_{\mathrm{e}}=1 \mathrm{~mm}\right.$ and $\left.N_{e}=8\right)$ yield computationally efficient solution. All finite element simulations presented in the current paper are based on the abovementioned optimum mesh size.

It can be seen in Figure 6 that the numerical solution obtained for the $\mathrm{C}-\mathrm{C}$ configuration model having artificially increased cohesive length and adjusted traction strength shows good agreement with the experimental results. Cohesive element deletion replicating mode-I crack growth happened approximately around the stated fracture energy material input of $0.65 \mathrm{~N} / \mathrm{mm}$.

3.4. DCB Results of C-M-C Configuration. Having validated the reliability of the developed finite element model to simulate mode-I crack growth, the fracture of adhesive interface between plastically deformed metal adherent and composite adherent was investigated using numerical approach. Experimental observation confirmed that no stiffness imbalance exists between the two dissimilar DCB arms, and complete crack growth is caused strictly by the opening mode of fracture. Figure 8 shows the load-displacement plots and the final permanently deformed shape of the fractured C-M-C configuration sample obtained from the experiments and the finite element model. With an increase in crack opening displacement, the nature of crack growth in experiments was found to change from stable to unstable manner. The continuous cumulative effect of plastic flow in metal layers makes the crack growth in a stick-slip manner in 


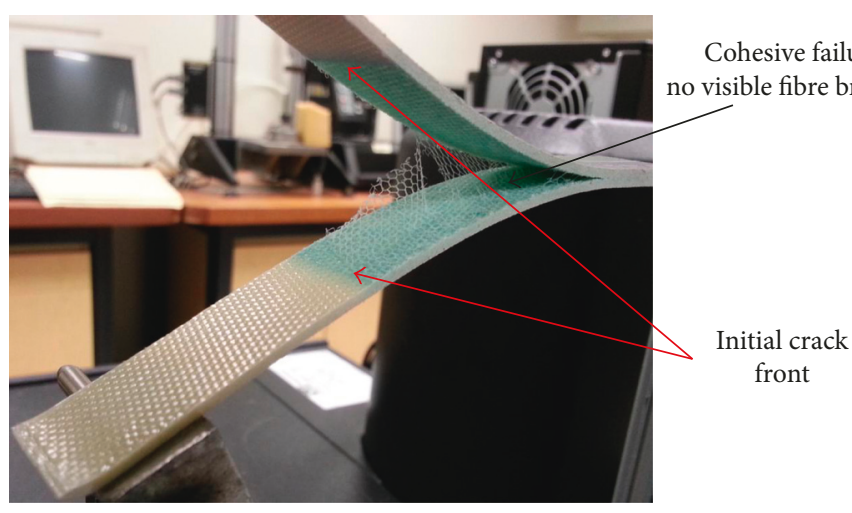

(a)

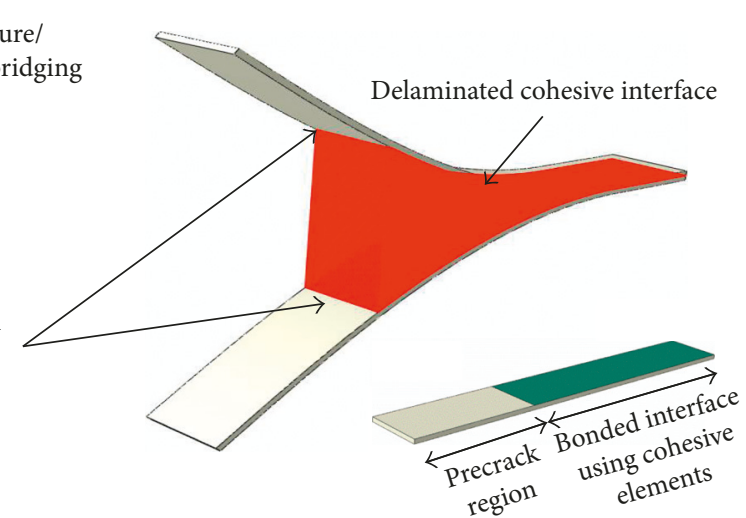

(b)

Figure 5: Mode-I fracture of C-C configuration: (a) experimental and (b) numerical.

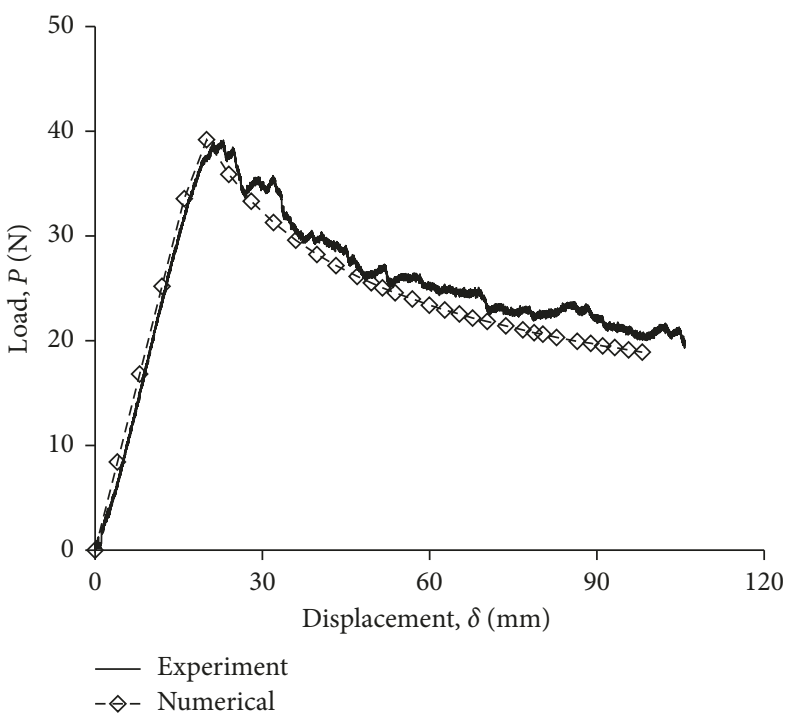

(a)

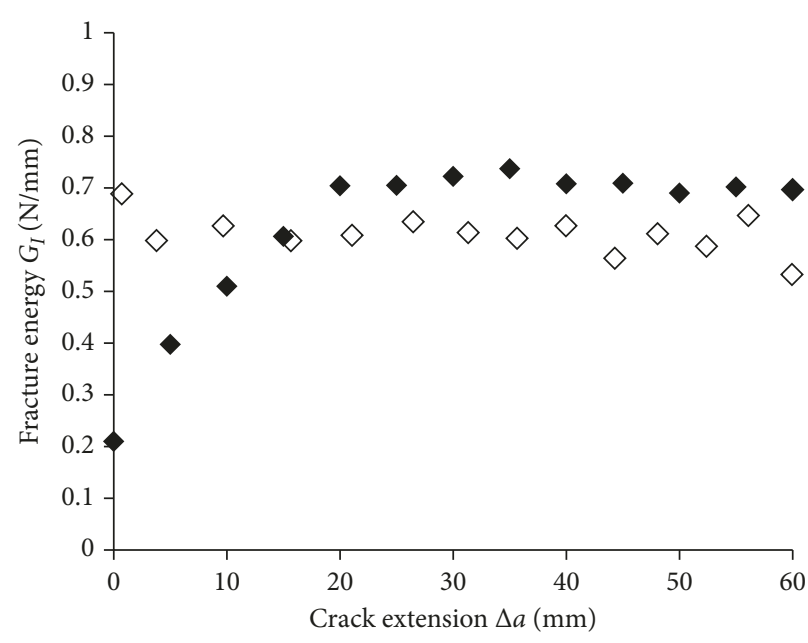

$\rightarrow$ Experiment

$\diamond$ Numerical

(b)

Figure 6: Finite element validation of the C-C configuration: (a) load-displacement curve and (b) fracture energy-crack extension.

TABLE 2: Adjusted normal interfacial strength with respect to different mesh sizes.

\begin{tabular}{ccccccc}
\hline$N_{e}$ & $l_{e}=1 \mathrm{~mm}$ & \multicolumn{2}{c}{$l_{e}=0.5 \mathrm{~mm}$} & \multicolumn{2}{c}{$l_{e}=0.3 \mathrm{~mm}$} & $\bar{t}_{\alpha}^{a}(\mathrm{MPa})$ \\
\hline 1 & $\bar{t}_{\alpha}^{a}(\mathrm{MPa})$ & $l_{\mathrm{cz}}(\mathrm{mm})$ & $\bar{t}_{\alpha}^{a}(\mathrm{MPa})$ & $l_{\mathrm{cz}}(\mathrm{mm})$ & 0.5 & 45.8 \\
3 & 25.5 & 1.0 & 36.1 & 1.5 & 26.4 & 0.3 \\
5 & 14.7 & 3.0 & 20.8 & 16.1 & 2.5 & 20.5 \\
8 & 11.4 & 5.0 & 12.8 & 4.0 & 1.5 \\
\hline
\end{tabular}

the form of periodic short bursts and crack arrest. This scenario leaves a combined cohesive and adhesive kind of fracture surface along the bonded adherent surfaces. Figure 9(a) shows the variation of the measured fracture energy $G_{I}$ using (2) against crack extension $\Delta a$; it can be seen that the plasticity effect causes $G_{I}$ to increase with increase in crack length. It seems that the peak propagation fracture energy increases nearly by 3.5 times more than the initiation fracture energy (i.e., $G_{I}$ at $\Delta a=0$ ).
The absence of a single value of $G_{I}$ from the experimental study makes the selection of an appropriate $G_{I}$ input data required for numerical simulation a very difficult one. The fact that the magnitude of the fracture energy of the adhesives is constant and independent of the adherent material and geometry when their failure surface along the adherent interface is completely cohesive [16]. Assuming that the simulated fracture surface of the C-M-C model is presumably cohesive, a propagation $G_{I}$ magnitude of 


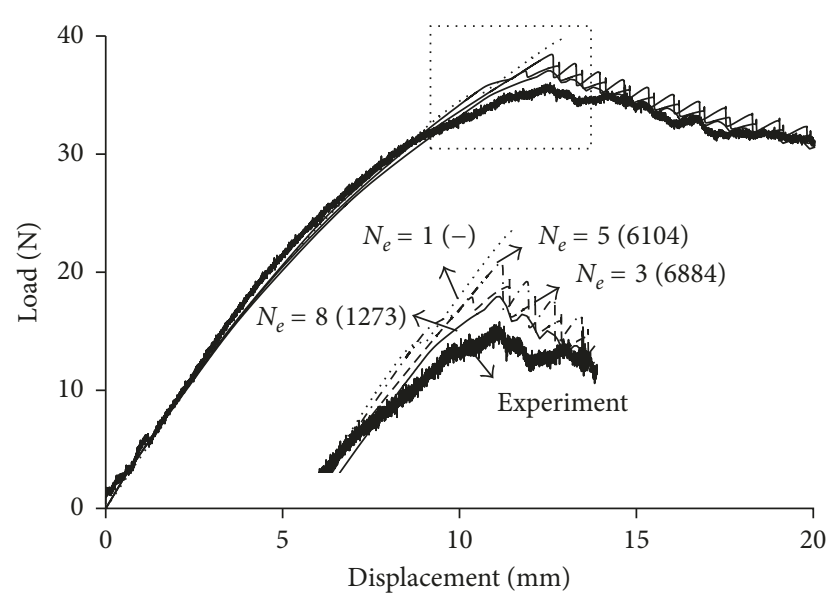

(a)

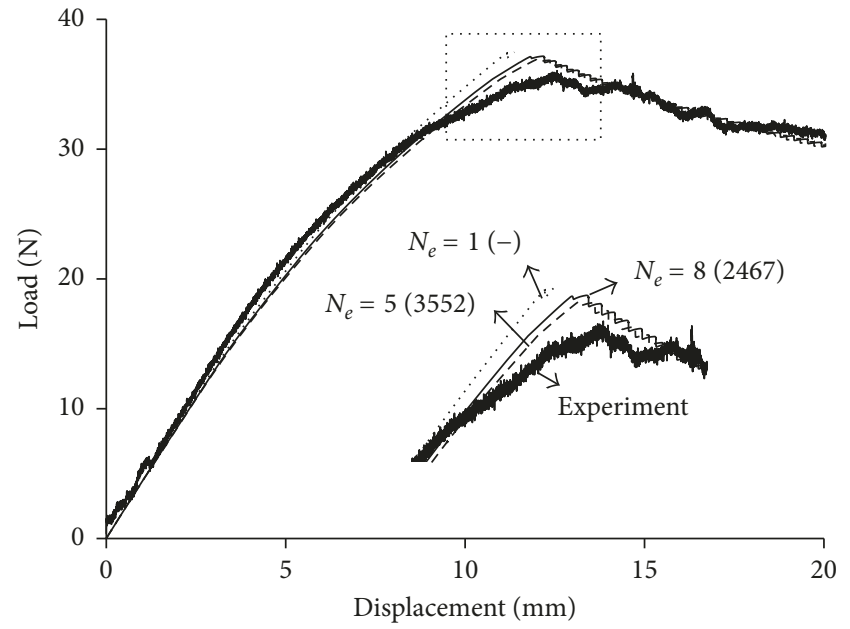

(b)

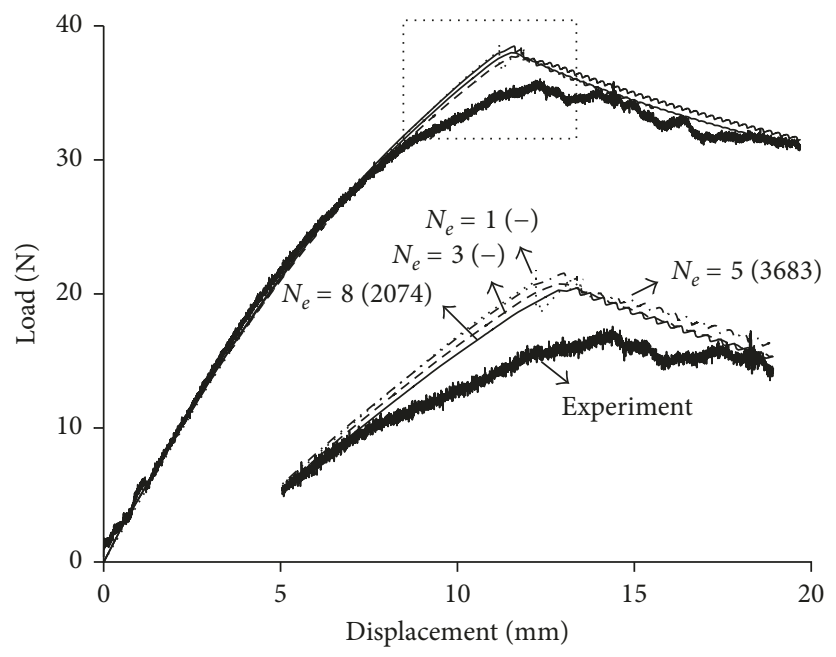

(c)

Figure 7: Load-displacement curve of C-C configuration for different cohesive element sizes: (a) $l_{e}=1 \mathrm{~mm}$, (b) $l_{e}=0.5 \mathrm{~mm}$, and (c) $l_{e}=0.3 \mathrm{~mm}$.

$0.65 \mathrm{~N} / \mathrm{mm}$ obtained in the monolithic C-C DCB sample is used instead. Two different numerical cases were simulated with the distinct material model definition for thin metal sheets: (i) pure elastic (El) and (ii) combined elastic-plastic (El-Pl) to investigate the influence of plastic effects. The material data required to model plastic response of metal layer were obtained from the standard tensile test as given in [17].

From Figure $8(\mathrm{a})$, it appears that the predicted initial elastic responses of both cases are correlated well with the experimental load-displacement curve. After reaching the plastic state, only the case with metal plasticity inclusion coincides with the experimental crack propagation phase until the stable crack region. Numerically, the plasticity effects were found to increase the propagation fracture energy nearly by an average of 1.5 times compared to the pure elastic model as shown in Figure 9(a).

The true fracture energy value was calculated by extracting the elastic strain energy magnitude from the fractured elastic-plastic numerical model and substituted into (4) which is similar to (3):

$$
\left(G_{I}\right)_{\mathrm{Tr}}=\frac{\Delta U_{R}}{B \Delta a}
$$

where $U_{R}$ is the recoverable (or elastic) strain energy obtained using ABAQUS output variable ALLSE in postprocessing.

By plotting $U_{R}$ versus $a$ as shown in Figure 9(b) and taking the derivative, the average true propagation fracture energy $\left(G_{I}\right)_{\operatorname{Tr}}$ can be found using (4). The values of the fracture energy obtained from the different cases are summarized in Table 3. The obtained $\left(G_{I}\right)_{\operatorname{Tr}}$ values are approximately equal to the initiation fracture energy $\left(G_{I}\right)_{\text {In }}$ predicted from experiment while it is $50 \%$ smaller than the average propagation fracture energy $\left(G_{I}\right)_{\mathrm{Pr}}$. Such huge differences are ultimately caused by the influence of the plasticity effect in the thin metal layer and its induced unstable nature of crack propagation.

\section{Conclusion}

The effect of adhesion between metal and composite layers on the mode-I fracture energy $G_{I}$ was investigated in the 


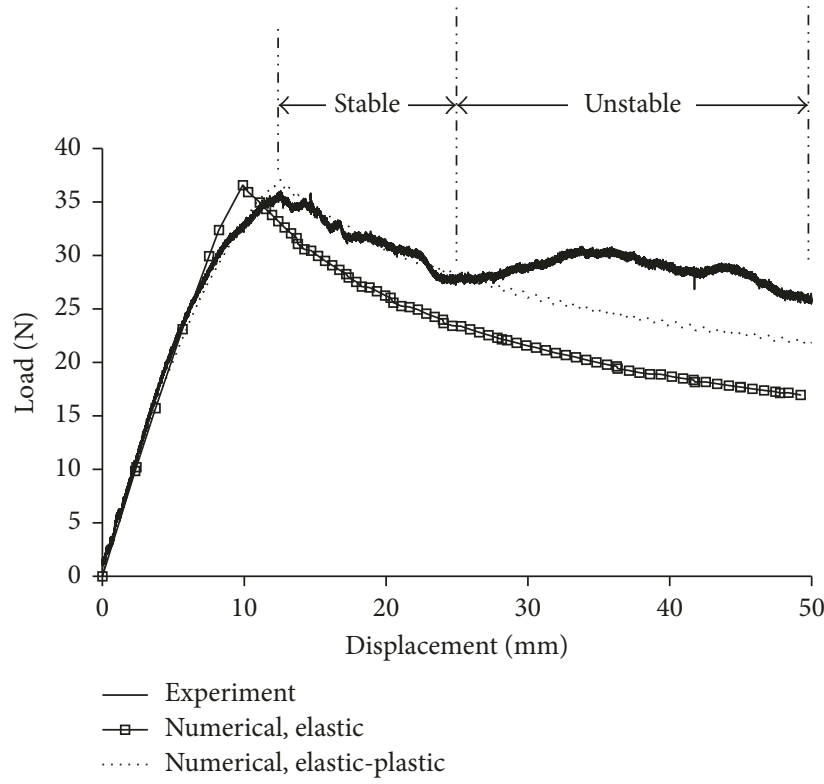

(a)

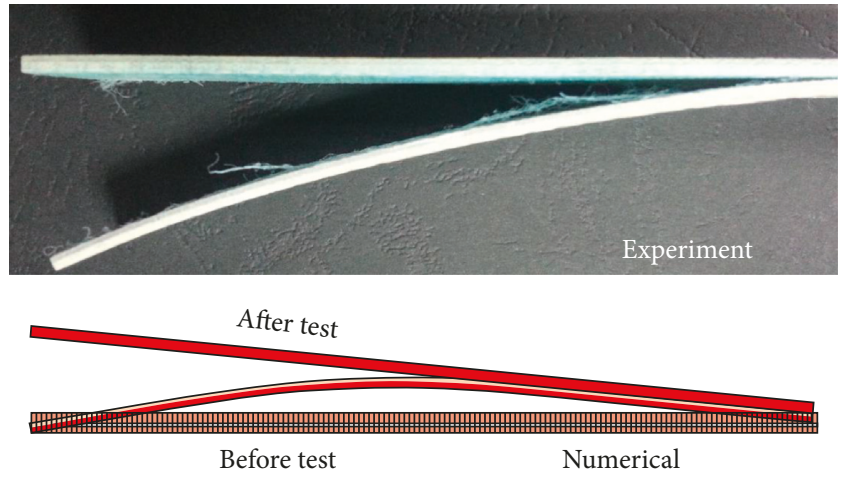

(b)

Figure 8: Mode-I fracture of the C-M-C configuration: (a) load-displacement curve and (b) permanent plastic deformation.

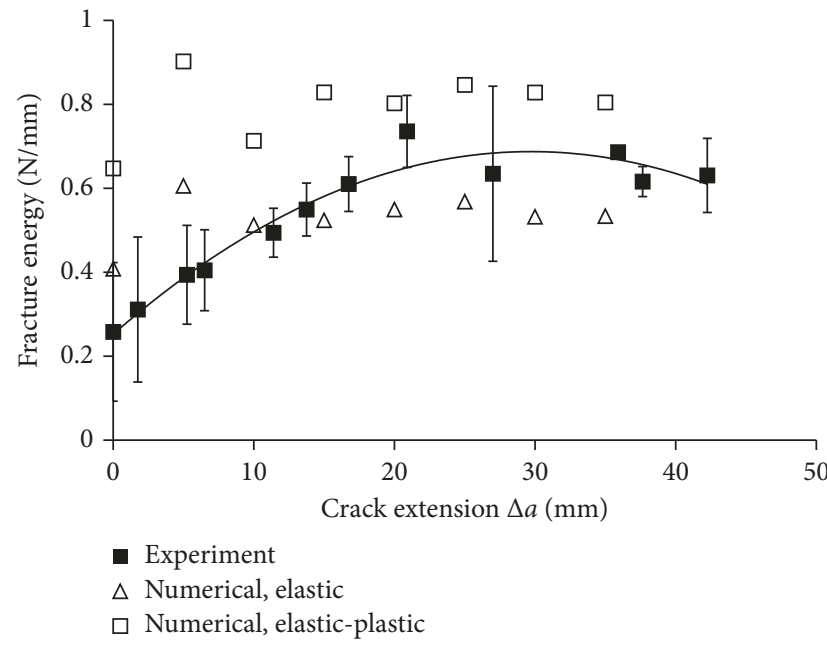

(a)

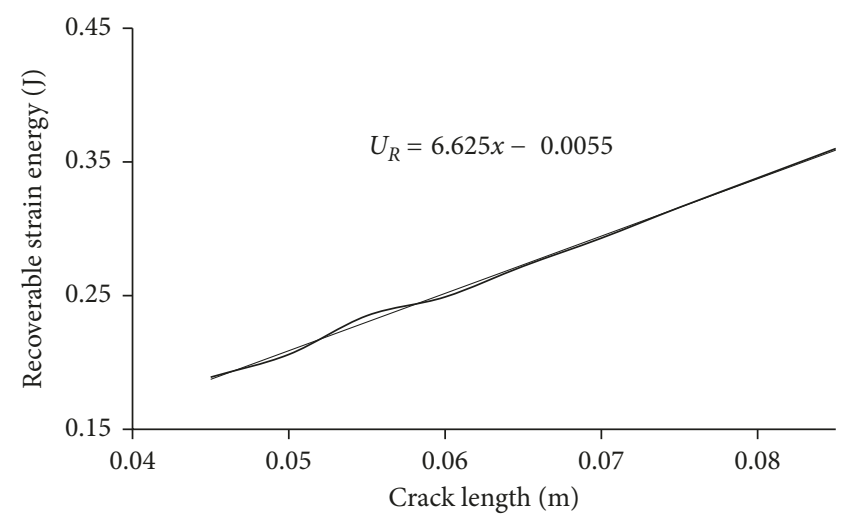

(b)

Figure 9: Mode-I fracture of C-M-C configuration: (a) fracture energy-crack extension and (b) recoverable elastic strain energy-crack length variation.

TABLE 3: Summary of fracture energy determined using C-M-C configuration.

\begin{tabular}{lcccc}
\hline Method & Experiment, using (1) & Numerical, elastic (3) & Numerical, elastic-plastic (3) & Numerical, elastic-plastic (4) \\
\hline Fracture energy $(\mathrm{N} / \mathrm{mm})$ & $\begin{array}{l}\left(G_{I}\right)_{\mathrm{In}}=0.257 \\
\left(G_{I}\right)_{\mathrm{Pr}}=0.595\end{array}$ & $\left(G_{I}\right)_{\mathrm{El}}=0.529$ & $\left(G_{I}\right)_{\mathrm{El}-\mathrm{Pl}}=0.796$ & $\left(G_{I}\right)_{\mathrm{Tr}}=0.265$ \\
\hline
\end{tabular}

fibre metal laminates (FMLs) using experimental and finite element study. A robust numerical approach was proposed to calculate the true fracture energy of the interface crack propagation between the plastically deformed metal and elastic composite surfaces. Different hybrid DCB configurations based on the type of adherends were used in the experimental study to observe metal-composite interface fracture not only in initiation phase but also in the complete propagation phase. The $\mathrm{M}-\mathrm{C}-\mathrm{M}$ configuration containing thick metal adherent resulted in highly unstable crack growth via nucleating multiple interfacial and interlaminar cracks, and no reliable fracture data can be extracted. 
An accurate fracture energy $G_{I}$ of the adhesive used was found using monolithic woven composite $\mathrm{C}-\mathrm{C}$ configuration. The crack propagation surfaces observed in the test were predominantly cohesive in nature, and no sign of fibre bridging was apparent. The obtained $G_{I}$ was used as a material input data in the developed finite element model to simulate damage evolution of interface cohesive element. Good correlation was found with the developed finite element model, and this ensured the reliability in modeling mode-I fracture in the interface of dissimilar layers.

Finally, the influence of the plastic deformation on $G_{I}$ was studied employing the C-M-C configuration where one arm of DCB adherent was designed to be metal-composite hybrid and the other with only composite material. This experimental study indicated that the plastic flow in thin metal layer continuously increases the measured $G_{I}$ by up to 3.5 times as the metal-composite interface fracture progresses. Similar response was also predicted in finite element study where the inclusion of the plastic constitutive model for metal layer increases the average propagation energy by 1.5 times compared to the pure elastic numerical model. A true fracture energy $\left(G_{I}\right)_{\operatorname{Tr}}$ was obtained using the numerically predicted internal elastic energy in the finite element model. The predicted result indicated that $\left(G_{I}\right)_{\mathrm{Tr}}$ was nearly equal to experimentally measured initiation fracture energy $\left(G_{I}\right)_{\text {In }}$ and $50 \%$ smaller than the average propagation fracture energy $\left(G_{I}\right)_{\mathrm{Pr}}$. This difference indicated the influence of metal plasticity effects on the measured $G_{I}$ magnitude through experiments.

\section{Appendix}

Cohesive zone length, $l_{\mathrm{cz}}$, defined as distance from crack front to the integration point of specified peak traction, $t_{\alpha}^{0}$, is expressed as

$$
l_{\mathrm{cz}}=\mathrm{ME} \frac{G_{\alpha}^{c}}{\left(t_{\alpha}^{0}\right)^{2}},
$$

and the number of cohesive elements, $N_{e}$, corresponding to given $l_{\mathrm{cz}}$ is expressed as

$$
N_{e}=\frac{l_{c z}}{l_{e}}
$$

where $E$ is Young's modulus of the interface material, $l_{e}$ is the cohesive element size, and $M$ is the constant cohesive parameter ranges between 0.2 and 1 . In the present work, the value of $M$ has been taken as 1 [18].

The minimum requirement of $N_{e}$ in $l_{c z}$ in order to accurately simulate the interface crack growth is not well established and ranges widely from 1 to 8 depending on the kind of bonded substrate material (DaVilla, Falk). For the case of FRP composite substrates, typical range of $l_{c z}$ is normally smaller than $1 \mathrm{~mm}$ and requires extremely fine mesh to simulate delamination. By combining (A.1) and (A.2), Turon [14] adjusted the magnitude of peak interface strength, $t_{\alpha}^{0}$, by changing the size and number of cohesive element. The equation for adjusted interface strength, $t_{\alpha}^{a}$, is shown in the following equation:

$$
t_{\alpha}^{a}=\sqrt{\frac{E G_{\alpha}^{c}}{N_{e} l_{e}}}
$$

\section{Conflicts of Interest}

The authors declare that they have no conflicts of interest.

\section{Acknowledgments}

The financial support in the form of a research student scholarship provided by the School of Mechanical and Aerospace Engineering, Nanyang Technological University, and the permission to use the laboratory and computing facilities at the School of Mechanical and Aerospace Engineering are truly acknowledged. It must also be mentioned that the fabrication and testing of the specimens by final year undergraduate students and postgraduate interns are acknowledged.

\section{References}

[1] A. Vlot and J. W. Gunnink, Fibre Metal Laminates: An Introduction, Kluwer Academic Publishers, Norwell, USA, 2001.

[2] G. B. Chai and P. Manikandan, "Low velocity impact response of fibre-metal laminates-A review," Composite Structures, vol. 107, pp. 363-381, 2014.

[3] M. Sadighi, R. C. Alderliesten, and R. Benedictus, "Impact resistance of fiber-metal laminates: a review," International Journal of Impact Engineering, vol. 49, pp. 77-90, 2012.

[4] T. Sinmazçelik, E. Avcu, M. Ö. Bora, and O. Çoban, "A review: fibre metal laminates, background, bonding types and applied test methods," Materials \& Design, vol. 32, no. 7, pp. 36713685, 2011.

[5] L. J. Hart Smith, "Adhesive joints for compositesphenomenological considerations," in Proceedings of Conference on Composite Technology, EI Segundo, CA, USA, September 1978.

[6] A. Vlot and J. W. Van Ingen, "Delamination resistance of post-stretched fibre metal laminates," Journal of Composite Materials, vol. 32, no. 19, pp. 1784-1805, 1998.

[7] B. A. Huppe, High Reliability Adhesive Joining of Metal and Composite Components, Department of Aeronautics and Astronautics, Massachusetts Institute of Technology, Cambridge, MA, USA, 2001.

[8] J. R. Reeder, K. Demarco, and K. S. Whitley, "The use of doubler reinforcement in delamination toughness testing," Composites Part A: Applied Science and Manufacturing, vol. 35, no. 11, pp. 1337-1344, 2004.

[9] M. D. Thouless, J. L. Adams, M. S. Kafkalidis, S. M. Ward, R. A. Dickie, and G. L. Westerbeek, "Determining the toughness of plastically deforming joints," Journal of Materials Science, vol. 33, no. 1, pp. 189-197, 1998.

[10] G. Lawcock, L. Ye, Y. W. Mai, and C. T. Sun, "The effect of adhesive bonding between aluminum and composite prepreg on the mechanical properties of carbon-fiber-reinforced metal laminates," Composites Science and Technology, vol. 57, no. 1, pp. 35-45, 1997.

[11] G. V. Reyes and W. J. Cantwell, "The mechanical properties of fiber-metal laminates glass fibre reinforced polypropylene," Composites Science and Technology, vol. 60, no. 7, pp. 10851094, 2000. 
[12] S. Mostovoy, E. J. Ripling, and C. F. Bersch, "Fracture toughness of adhesive joints," Journal of Adhesion, vol. 3, no. 2, pp. 125-144, 1971.

[13] K. Song, K. C. G. Dávila, and C. A. Rose, "Guidelines and parameter selection for the simulation of progressive delamination," in Proceedings of Abaqus User Conference, Newport, RI, USA, May 2008.

[14] A. Turon, C. G. Dávila, P. P. Camanho, and J. Costa, "An engineering solution for mesh size effects in the simulation of delamination using cohesive zone models," Engineering Fracture Mechanics, vol. 74, no. 10, pp. 1665-1682, 2007.

[15] B. R. K Blackman, J. P. Dear, A. J. Kinloch, and S. Osiyemi, "The calculation of adhesive fracture energies from doublecantilever beam test specimens," Journal of Materials Science Letters, vol. 10, no. 5, pp. 253-256, 1991.

[16] B. R. K Blackman, A. J. Kinloch, F. S. Rodriguez-Sanchez, and W. S. Teo, "The fracture behaviour of adhesively-bonded composite joints: effects of rate of test and mode of loading," International Journal of Solids and Structures, vol. 49, no. 13, pp. 1434-1452, 2012.

[17] P. Manikandan and G. B. Chai, "A layer-wise behavioral study of metal based interply hybrid composites under low velocity impact load," Composite Structures, vol. 117, pp. 17-31, 2014.

[18] A. Hillerborg, M. Modéer, and P. E. Petersson, "Analysis of crack formation and crack growth in concrete by means of fracture mechanics and finite elements," Cement and Concrete Research, vol. 6, no. 6, pp. 773-781, 1976. 


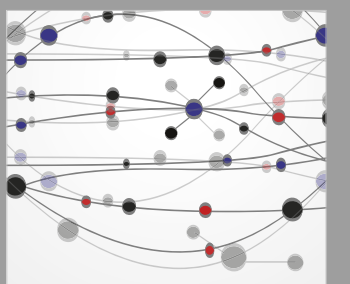

The Scientific World Journal
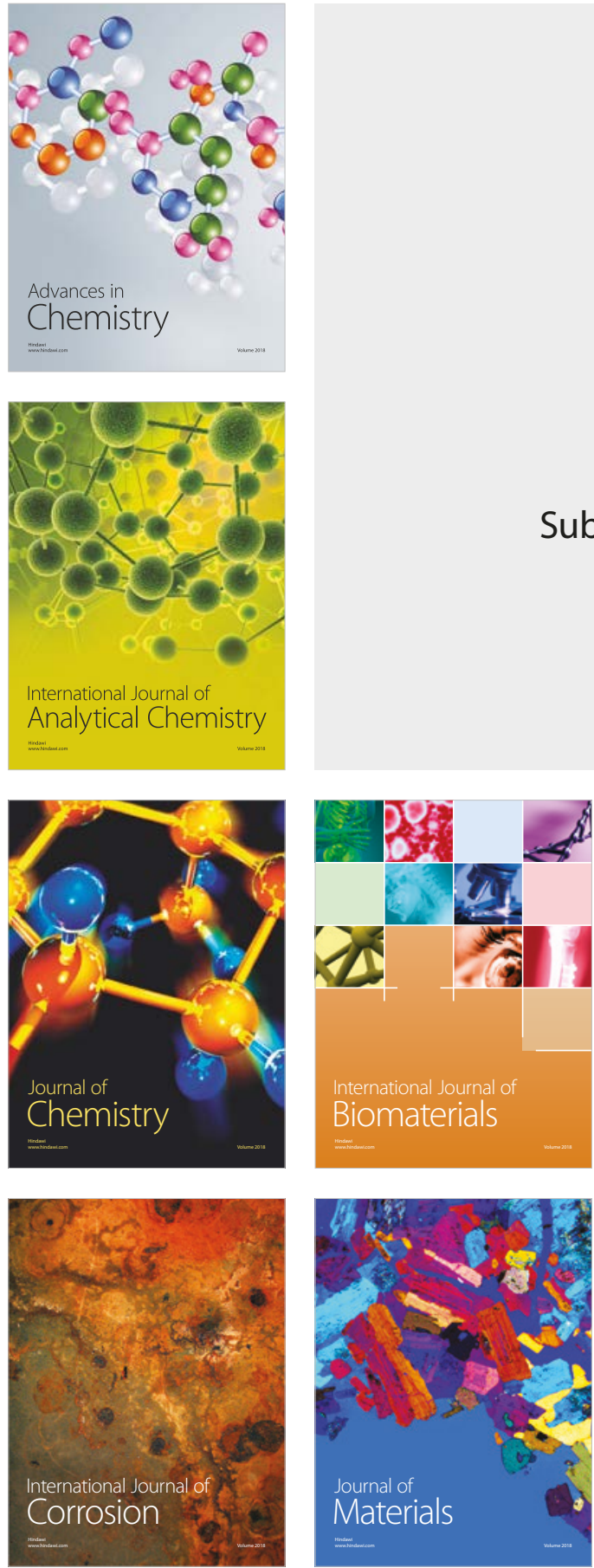

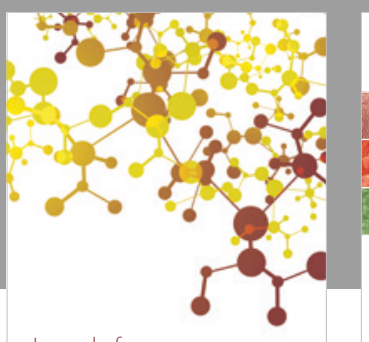

Journal of

Applied Chemistry
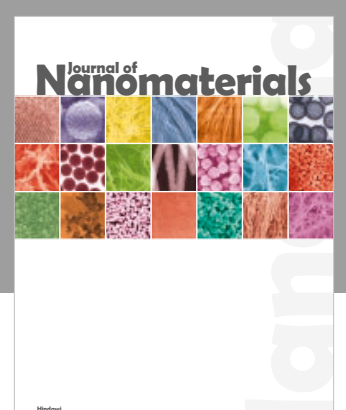

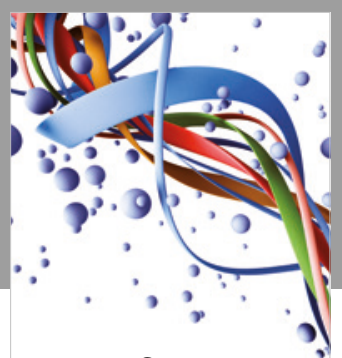

Scientifica

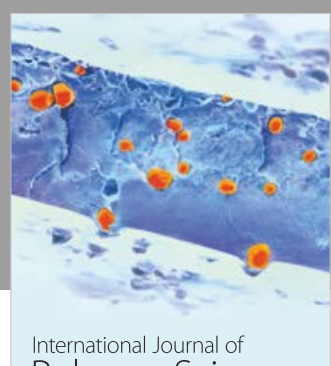

Polymer Science

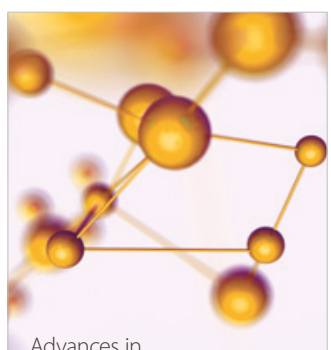

Physical Chemistry
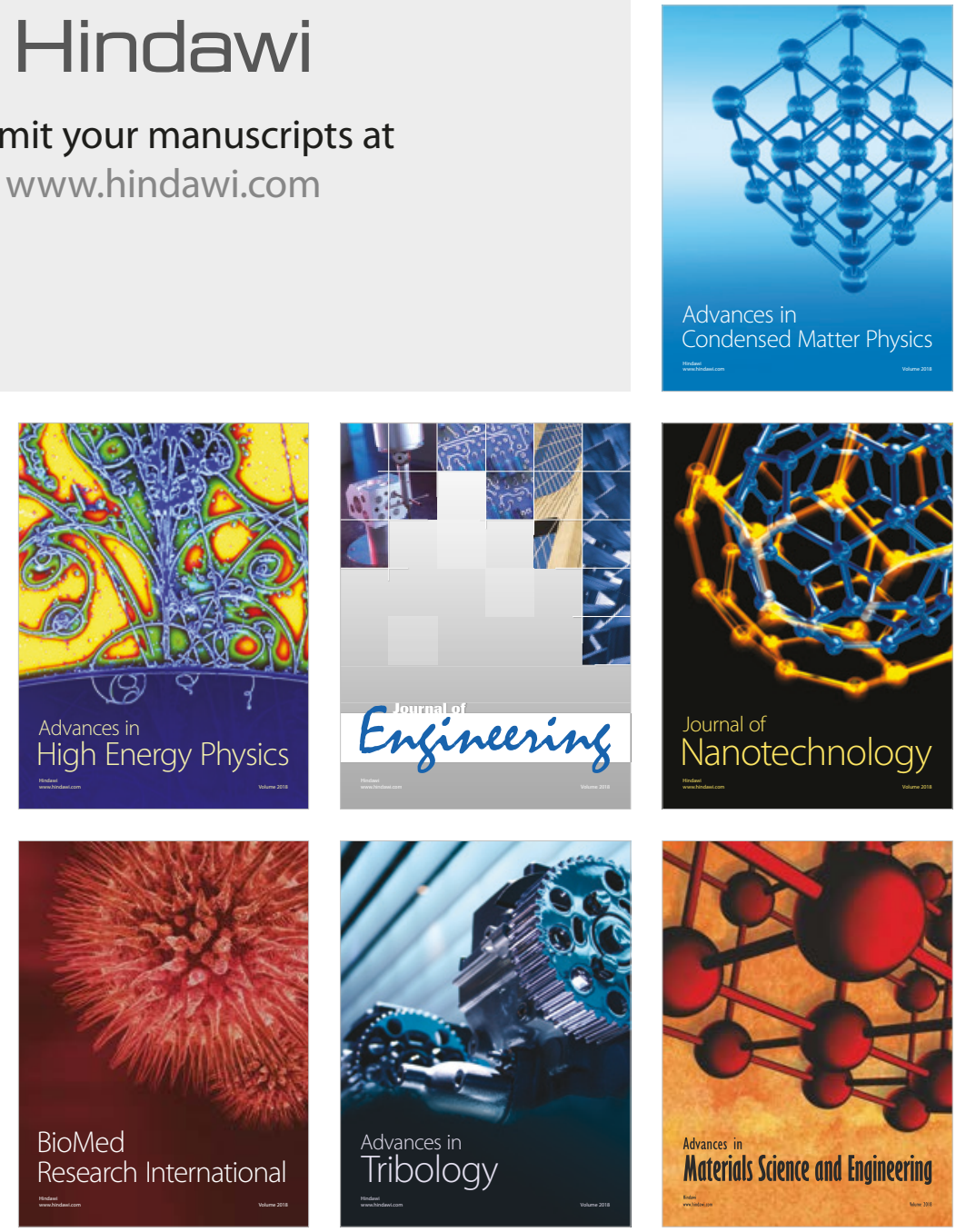